\title{
In silico Activity Analysis of Saponins and 2, 5-Piperazinedione from Marine Organism against Murine Double Minute-2 Inhibitor and Procaspase-3 Activator
}

\author{
Arry Yanuar, Indah Pratiwi, Rezi Riadhi Syahdi* \\ Faculty of Pharmacy, Universitas Indonesia, Depok 16424 West Java, INDONESIA.
}

\begin{abstract}
Objective: Cancer is a disease that can occur because of apoptosis failure. One of the causes of apoptosis failure is the presence of MDM2 inhibiting the activity of p53 so procaspase-3 could not be activated to caspase-3. Currently, the treatment of cancer has been applied widely, but more effective treatment always needed because cancer has been mutated. One of the treatments is the search for natural resources that come from the sea. The pursuit of the bioactive compound from marine organisms is not straightforward because it takes a long time and costly. Methods: Therefore, in silico method is used. This study conducted a docking of bioactive compounds from saponin and 2, 5-Piperazinedione as the MDM2 inhibitor and procaspase-3 activator by using Auto Dock and Vina. Results: The results showed that 18-Oxotryprostatin A and Intercedenside $A$ were the best bioactive compounds to serve as MDM2 Inhibitor and 6-Methoxyspirotryprostatin $B$ and Frondoside $A$ as a procaspase-3 activator due to their low binding energy. Conclusion: Most recommended bioactive
\end{abstract}

compounds are those who have low binding energy, which is 18-Oxotryprostatin $A$ with the value of $-8,6 \mathrm{kcal} / \mathrm{mol}$ and $I$ tercedenside $A$ with the value of $-7,0 \mathrm{kcal} / \mathrm{mol}$.

Key words: 2,5-Piperazinedione, Activator procaspase-3, Inhibitor MDM2, Cancer, Molecular docking, Saponin.

\section{Correspondence}

Rezi Riadhi Syahdi, Faculty of Pharmacy, Universitas Indonesia, Depok 16424 West Java, INDONESIA.

Phone: +6287878588772

Email: rezi.riadhi@farmasi.ui.ac.id, icapps2017.farmasiui@gmail.com

DOI: 10.5530/jyp.2018.2s.4

\section{INTRODUCTION}

Cancer may occur due to mutations caused by the lack of p53 activity. ${ }^{1}$ The p53 is a positive regulator which plays a role in regulating the process of apoptosis. In the abnormal condition (cancer), p53 will bind to MDM2 a negative regulator, regulating the activity of $\mathrm{p} 53$ by inhibiting it. When p53 is activated, the amount of MDM2 will increase, and MDM2 will inhibit the activity of p53, causing apoptosis failure. ${ }^{2}$ Inhibition of p53 activity may interfere intrinsic and extrinsic pathways in apoptosis to be inhibited so procaspase- 3 could not be activated to caspase-3, an executing agent of apoptosis in damaged cells. Cells with caspase- 3 deficiency will lead to cancer. The effective treatment is needed because cancer has been mutated. One of the treatments is the search for natural resources that come from the sea. Saponins and 2, 5-Piperazinedione are candidate drugs from marine organisms to use in this study. Previous studies showed that saponins are cytotoxic toward cancer cells and some mechanisms of action such as cell cycle progress, induced apoptosis, and microtubule stabilization. ${ }^{3}$ In saponins a series of bioactive compounds that have an anticancer activity such as Intercedenside A and B, Frondoside A, and Philinopside A isolated from marine sea cucumber organisms. The 2,5-Piperazinedione has become the primary focus in many studies due to its biological activity, such as it is cytotoxic activity in cancer cells, antimicrobials and anti-inflammatory. ${ }^{4}$ Some of the 2, 5-Piperazinedione bioactive compounds that showed anti-cancer activity had been isolated from marine fungi, i.e., Gliocladride B, 18-Oxotryprostatin A, 6-Methoxyspirotryprostatin B and Spirogliotoxin.

The search of a bioactive compound from marine organisms is expensive and time-consuming. Therefore, in silico method was used. In silico study could minimize the failure that possibilities in tests conducted in vivo or in vitro, by predicting failure possibilities such as the interaction between a compounds with target receptors. ${ }^{5}$

\section{MATERIALS AND METHODS}

\section{Hardware}

A server computer With following specifications: processor (CPU) Intel $^{\circledR}$ Xeon E5620, graphics processing unit (GPU) Nvidia ${ }^{\star}$ GeForce GTX 780, dan 32GB Random Access Memory (RAM) DDR3 with Linux Ubuntu 12.04 LTS was used. Two client computers with the following specification was used. The first computer was Lenovo Ideapad $310 \mathrm{~S}$ (Lenovo ${ }^{\circledR}$, China), Intel Celeron N3350 dual-core computer processing unit (CPU), and 2GB RAM DDR3L with Microsoft Windows 10 64-bit operating system. The second computer specification was Lenovo Ideapad Z410 with Intel ${ }^{\oplus}$ Core $^{\mathrm{Tx}}$ i5-4200M computer processing unit (CPU), GPU Nvidia ${ }^{\star}$ GeForce $^{\circledR}$ GT 740M, 8GB RAM DDR3L with Microsoft Windows 8 Pro 64-bit operating system and Linux Ubuntu 12.04 LTS.

\section{Software}

The software used in this research are PyMOL (DeLano Scientific LLC, Italia), AutoDockTools (The Scripps Research Institute, USA), PuTTY), LigandScout (InteLigand', Austria), UCSF Chimera (University of California, San Fransisco), MarvinSketch (ChemAxon, Budapest), LigPlot (EMBLEBI, UK), Pose View, dan PyRx (The Scripps Research Institute, USA). 


\section{D Structure}

The saponins and 2, 5-Piperazinedione, macromolecule compounds such as MDM2 (5LN2) and Procaspase-3 (4JR0), and positive control compound.

\section{Preparation of macromolecule structure}

The preparation process was consisted of searching and downloading, optimization, separation of non-standard residues and determination of binding sites.

\section{Preparation of ligand 3D structure}

There were three types of ligands used for docking, i.e. (1) Ligands derived from separated macromolecules; (2) Marine bioactive compounds available in ChemSpider Search and Share Chemistry (http://ChemSpider.com), PubChem Open Chemistry Database (http://PubChem.ncbi.nlm.gov), and other sources; (3) Positive controls obtained from literature studies which activities had been recognized by the FDA for cancer treatment and available in the PubChem Open Chemistry Database (http://PubChem.ncbi.nlm.gov) and ChemSpider.

\section{Optimization and Validation of Molecular Docking Parameter}

The validation of molecular docking method was conducted by redocking the crystals that had been separated from macromolecules. The dimension size (grid box) parameters of the crystal ligand were varied. The corresponding dimension size (grid box) parameter produced docking results having interaction with similar amino acids, low RMSD values $(\leq 2.0 \AA)$, more negative free binding energy values and more homogeneous cluster distributions. In each parameter, the implementation of running GA 25 times for the use of Autodock Tools software in the dpf file for AutoDock 4. While on AutoDock Vina, the exhaustiveness value was 8 .

\section{Molecular Docking of Positive Control Compound}

The docking of positive control compounds was performed to obtain the score of each active compound used for docking. The positive controls used were amg232, rg7112, compound 1541 and compound 42 . The four selected positive controls were obtained from accessible literature studies on PubChem.

Docking of positive control against procaspase- 3 macromolecule was conducted by blind docking. Blind docking is a method to detect a possibility of binding sites and peptide ligands modes by scanning the entire surface of the protein target. ${ }^{6}$ Blind docking can be performed by increasing the scale of the grid box dimensions to cover the whole macromolecule.

The docking of positive control on MDM2 macromolecule was conducted by molecular docking method.

\section{Molecular Docking of Marine Organism Compound}

Molecular docking was performed by using AutoDock4 and Vina. The coordinates of the binding site and the grid box has used the value based on the redocking results of each macromolecule crystal. AutoDock 4 was run with set parameters: number generation algorithm 27.000 , calculate 2.500 .000 times (Medium), population 150. In molecular docking by Vina, exhaustiveness was set to 8 (short). The results of molecular docking by using Vina showed the best ligand conformation in the ${ }^{*}$. pdbqt file format.

\section{Analysis and Visualization of Protein-Ligand Interaction}

The results of docking by using AutoDock 4 and AutoDock Vina were stored as ${ }^{*}$.pdb. Docking by using AutoDock Vina was conducted by the separation of docking results using VinaSplit. The visualization of docking results was performed by using LigandScout, LigPlot dan PyMOL software.

The purpose of the analysis and visualization was to analyze the presence of hydrogen bond, hydrophobic interaction between ligand compound and positive control to each targetted receptor based on the parameter used in AutoDock and Vina.

\section{RESULTS}

The separated macromolecule and ligand files in ${ }^{*}$.pdb format were converted to ${ }^{*}$.pdbqt by using AutoDockTools. The coordinates of each ligand were determined as coordinates of the binding site used in docking. Determination of coordinates for the receptor target was performed by using a grid box search method concentrating on the crystal ligands. The result is shown in Table 1.

The optimization of the molecular docking method was performed by redocking the ligand of previously separated macromolecules. The docking was conducted to prove that the selected docking parameters in docking method are valid. ${ }^{7}$ The macromolecules used for re-docking was an MDM2 inhibitor. Re-docking was performed by using a co-crystal ligand against macromolecules. The results showed that the docking parameters had more negative binding free energy value, a more homogeneous cluster distribution, and RMSD (Root Mean Square Deviation) with a value of $\leq 2,0 \AA$.

In the case of procaspase- 3 activator, blind docking method was performed because the binding site or docking location was not detected. Blind docking was performed with positive control as the ligand by using a $126 \mathrm{x}$ $126 \times 126$ unit's grid box to cover the entire macromolecules.

The re-docking of MDM2 inhibitor was performed by using AutoDock Tools and Vina software. The parameters used were the number of algorithm generation 27.000, population 150, calculations 2.500 .000 times (medium), and the implementation of running GA as much as 25 times for the use of AutoDock Tools software. For Vina parameter, the value of exhaustiveness is 8 . In blind docking of procaspase- 3 activator, the AutoDock Tools software used the algorithm generation parameter of 27.000 , the population 150, the calculation of 2.500 .000 (medium), and the implementation of running GA as much as 100 times. While on Vina, the exhaustiveness value is 8 .

For procaspase- 3 , the positive control that has the best docking parameter is compound 1541 with the lowest binding free energy of $-10,4 \mathrm{kcal} / \mathrm{mol}$ and grid box $26,250 \AA$. The best parameter compound 1541 was obtained by using Vina. While in macromolecule MDM2, positive control compound which has the lowest binding free energy $-10,48 \mathrm{kcal} / \mathrm{mol}$ is AMG 232 and grid box 40x40x40 unit. The best parameter for AMG232 was obtained by using AutoDock.

Based on the docking results, it could be concluded that the lowest binding free energy value in the marine bioactive compound was produced by using Vina parameter, while in AutoDock the binding free energy of the resulting is not as good as Vina. This bad result is due to AutoDock limitation on running the program correctly when the modeled structure has poor quality, or too much torsion when the maximum torsion on AutoDock is $32 .{ }^{8}$

\section{Table 1: Coordinates $(x, y, z)$ on the target macromolecules.}

\begin{tabular}{ccccc}
\hline No & Macromolecule & $\mathbf{X}$ & $\mathrm{Y}$ & $\mathrm{Z}$ \\
\hline 1 & $5 \mathrm{LN} 2$ & -9.442 & -10.504 & 0.238 \\
2 & 4JR0 & 5.333 & 6.773 & 11.972 \\
\hline
\end{tabular}




\section{DISCUSSION}

The optimization results by using AutoDock and Vina showed that the optimal grid box for MDM2 inhibitor was $40 \times 40 \times 40$ showing lowest free binding energy value. This study involved bioactive compounds that have a large number of molecules; therefore, the $70 \times 70 \times 70$ grid box was still used because it is expected to produce the lowest free binding energy. The $70 \times 70 \times 70$ grid box obtained by doing blind docking was the optimal grid box for the procaspase- 3 activator. The validation of molecular docking was conducted by calculating the RMSD value manually using PyMOL. This RMSD value was used as a reference to see the docking results regarding the position similarity to co-crystal ligand before it is separated. RMSD value of AutoDock and Vina parameter is shown in Table 2.

Therefore, the visualization results by using Pose View showed that redocking results have similarity with the original interaction on the crystal (5LN2) located on the gridbox 40x40x40 where the amino acid residues produced have similarity with the original PDB crystals before redocking consisting of hydrophobic interactions on the amino acid residues of Met26A, Gly58A, Leu54A, Ile61A, His96A, Ile99A and Val93A and hydrogen bonds acceptor with His96A residue.

The Vina parameter is used as the best comparator of the validation result. Vina Autodock runs with dimensions (gridbox) $40 \times 40 \times 40$ and $70 \times 70 \times 70$ unit, gridbox value is equalized to angstrom of 15,000 and 26,250 because 1 unit is equal to 0.375 angstrom and exhautiveness is set at 8 . The validation of the Vina parameter is the same as the Autodock parameter by calculating the RMSD (Root Mean Square Deviation) value manually using PyMOL.
Table 2: RMSD value of (A) AutoDock parameter and (B) Vina parameter.

A

\begin{tabular}{|c|c|c|}
\hline No & Grid Box (Unit) & $\operatorname{RMSD}(\AA ̊)$ \\
\hline 1 & $40 \times 40 \times 40$ & 1.36 \\
\hline 2 & $70 \times 70 \times 70$ & 1.72 \\
\hline \multicolumn{3}{|c|}{ B } \\
\hline No & Grid Box (Å) & RMSD (Å) \\
\hline 1 & 15.000 & 1.41 \\
\hline 2 & 26.250 & 1.75 \\
\hline
\end{tabular}

From the redocking of ligands to macromolecules, the optimal docking parameters are generated from the 40x40x40 gridbox with low free binding energy values and qualified RMSD values. The result of free binding energy value obtained is $-9.2 \mathrm{kcal} / \mathrm{mol}$ with RMSD value obtained manually using PyMOL that is equal to $1,406 \AA$ and the inhibition constant obtained by using the formula below is $178 \mathrm{nM}$ ( $\Delta \mathrm{G}=\mathrm{RT} \operatorname{lnK})$.

The best compound obtained from 2,5-Piperazinedione were 18-Oxotryprostatin A with binding free energy value $-8,6 \mathrm{kcal} / \mathrm{mol}$ as an MDM2 inhibitor and 6-Methoxyspirotryprostatin B with binding free energy value $-9,6 \mathrm{kcal} / \mathrm{mol}$ as a procaspase- 3 activator. While in saponins, the compound that provides lowest binding free energy is Intercedensides A with value $-7,0 \mathrm{kcal} / \mathrm{mol}$ as an MDM2 inhibitor and Frondoside A with value $-9,2 \mathrm{kcal} / \mathrm{mol}$ as a procaspase- 3 .

The Protein-ligand interaction was analyzed and visualized by using LigandScout, LigPlot, and PyMOL ${ }^{9-11}$ to inspect the hydrogen bond distance and hydrophobic interactions (Table 3 and 4).

Table 3: Interaction analysis of marine bioactive compounds against MDM2 inhibitors using LigandScout, LigPlot, and PyMOL.

\begin{tabular}{|c|c|c|c|c|}
\hline \multirow[t]{2}{*}{ Compound } & \multicolumn{2}{|c|}{ Amino Acid Residue Interaction } & \multirow{2}{*}{$\begin{array}{c}\text { Binding Energy } / \Delta \mathrm{G} \\
\text { (kcal/mol) } \\
\text { Autodock }\end{array}$} & \multirow[b]{2}{*}{ Vina } \\
\hline & Autodock & Vina & & \\
\hline 6-Methoxyspirotryprostatin B & $\begin{array}{c}\text { Leu54, Gly58, Phe91, } \\
\text { Val93, } \\
\text { His96, } \\
\text { Ile99 }\end{array}$ & $\begin{array}{l}\text { Leu54, Gly58, Met62, Val93, His96, } \\
\text { Ile99 }\end{array}$ & -7.81 & -7.2 \\
\hline 18-Oxotyprostatin A & $\begin{array}{c}\text { Leu54, Gln59, Phe91, Val93, His96, } \\
\text { Ile99 }\end{array}$ & $\begin{array}{c}\text { Leu54, Gly58, Val93, His96, Ile99, } \\
\text { Tyr100 }\end{array}$ & -8.66 & -8.6 \\
\hline Gliocladride B & $\begin{array}{c}\text { Leu54, Gly58, Gln59, Val93, } \\
\text { His96 } \\
\text { Ile99, }\end{array}$ & Leu54, Gly58, His96, Ile99, Tyr100 & -7.67 & -7.4 \\
\hline Spirogliotoxin & $\begin{array}{c}\text { Leu54, Gly58, Gln59, Phe91, Val93, } \\
\text { His96 } \\
\text { Ile99, }\end{array}$ & Leu54, Gly58, Met62, Val93, Ile99 & -6.34 & -6.2 \\
\hline Frondoside A & $\begin{array}{c}\text { Leu54, Gly58, Gln59, Va193, Phe91, } \\
\text { His96, } \\
\text { Ile99 }\end{array}$ & $\begin{array}{c}\text { Leu54, Gly58, Met62, Val93, His96, } \\
\text { Tyr100 }\end{array}$ & -4.12 & -6.8 \\
\hline Intercedenside A & Leu54, Gly58, Gln59, Val93, & $\begin{array}{l}\text { Leu54, Gly58, Met62, Val93, His96, } \\
\text { Ile99 }\end{array}$ & -5.11 & -7 \\
\hline Intercedenside B & Leu54, Gln59, Val93, His96, & $\begin{array}{c}\text { Leu54, Gly58, Val93, His96, Met62, } \\
\text { Tyr100 }\end{array}$ & -6.13 & -6.2 \\
\hline Philinopside A & Leu54, Gly58, Val93, His96 & $\begin{array}{c}\text { Leu54, Gly58, Met62, Val93, His96, } \\
\text { Tyr100 }\end{array}$ & -5.62 & -6.8 \\
\hline
\end{tabular}


Yanuar, et al.: In silico MDM2 Inhibitor and Procasp-3 Activator Analysis of Saponins and 2,5-Piperazindions

Table 4: Interaction analysis of marine bioactive compounds against procaspase-3 activator using LigandScout, LigPlot, and PyMOL.

\begin{tabular}{|c|c|c|c|c|}
\hline \multirow[t]{2}{*}{ Compound } & \multicolumn{2}{|c|}{ Amino Acid Residue Interaction } & \multicolumn{2}{|c|}{ Binding Energy $/ \Delta \mathrm{G}(\mathrm{kcal} / \mathrm{mol})$} \\
\hline & Autodock & Vina & Autodock & Vina \\
\hline 6-Methoxyspirotryprostatin B & $\begin{array}{c}\text { Glu124, Gly125, Leu136, Lys137, Tyr197, } \\
\text { Pro201, Val266 }\end{array}$ & $\begin{array}{l}\text { Glu124, Gly125, Lys137, Arg164, } \\
\text { Tyr195, Tyr197, Val266 }\end{array}$ & $-8,49$ & $-9,6$ \\
\hline 18-Oxotyprostatin A & $\begin{array}{l}\text { Glu124, Gly125, Leu136, Lys137, Tyr197, } \\
\text { Pro201, Val266 }\end{array}$ & $\begin{array}{l}\text { Glu124, Gly125, Lys137, Arg164, } \\
\text { Tyr195, Tyr197, Val266 }\end{array}$ & $-8,09$ & $-8,6$ \\
\hline Gliocladride B & $\begin{array}{c}\text { Gly125, Leu136, Lys137, Tyr197, Pro201, } \\
\text { Val266 }\end{array}$ & $\begin{array}{l}\text { Gly125, Lys137, Arg164, Tyr195, } \\
\text { Tyr197, Val266 }\end{array}$ & $-8,15$ & $-8,9$ \\
\hline Spirogliotoxin & $\begin{array}{l}\text { Gly125, Leu136, Lys137, Tyr197, Pro201, } \\
\text { Val266 }\end{array}$ & $\begin{array}{l}\text { Gly125, Lys137, Arg164, } \\
\text { Tyr195, Tyr197, Val266 }\end{array}$ & $-6,43$ & $-6,9$ \\
\hline Frondoside A & Glu124, Gly125, Leu136, Lys137, Pro201 & $\begin{array}{l}\text { Glu124, Lys137, Arg164, } \\
\text { Tyr195, Tyr197, Val266 }\end{array}$ & $-4,05$ & $-9,2$ \\
\hline Intercedenside A & $\begin{array}{l}\text { Glu124, Gly125, Lys137, Tyr197, Pro201, } \\
\text { Val266 }\end{array}$ & $\begin{array}{l}\text { Glu124, Gly125, Lys137, Arg164, } \\
\text { Tyr195, Tyr197, Val266 }\end{array}$ & $-6,54$ & $-8,9$ \\
\hline Intercedenside B & Glu124, Gly125, Lys137 & $\begin{array}{c}\text { Glu124, Lys137, Arg164, Tyr195, } \\
\text { Tyr197 }\end{array}$ & $-5,92$ & $-8,5$ \\
\hline Philinopside A & $\begin{array}{l}\text { Glu124, Gly125, Leu136, Lys137, Tyr197, } \\
\text { Pro201 }\end{array}$ & $\begin{array}{l}\text { Glu124, Gly125, Lys137, Arg164, } \\
\text { Tyr195, Tyr197, Val266 }\end{array}$ & $-6,80$ & $-8,6$ \\
\hline
\end{tabular}

\section{CONCLUSION}

Based on the validation result of molecular docking, the parameter that showed low free binding energy $(\Delta \mathrm{G})$ by using AutoDock Vina software with grid-box value is $26,250 \AA$.

The best compound obtained from 2,5-Piperazinedione with free low binding energy were 18-Oxotryprostatin A as an MDM2 inhibitor with the value is $-8,6 \mathrm{kcal} / \mathrm{mol}$ and 6-Methoxyspirotryprostatin $\mathrm{B}$ as a procaspase- 3 activator with the value is $-9,6 \mathrm{kcal} / \mathrm{mol}$. While in saponins, the compounds that provide low free bonding energy $(\Delta \mathrm{G})$ were Intercedenside $\mathrm{A}$ as an MDM2 inhibitor with the value of $-7,0 \mathrm{kcal} / \mathrm{mol}$ and Frondoside A as a Procaspase- 3 activator with the value of $-9,2 \mathrm{kcal} / \mathrm{mol}$.

\section{ACKNOWLEDGEMENT}

Author thanks to Universitas Indonesia for funding to this research through PITTA 2017.

\section{CONFLICT OF INTEREST}

The authors hereby declare that they have no affiliations with organization, project or entity with commercial purposes or financial interest or without financial interest in which the subject or materials addressed in the manuscript

\section{ABBREVIATIONS}

p53: Tumor protein 53, phosphoprotein 53; MDM2: Murine double minute-2; FDA: United States Food and Drug Administration; CPU:
Computer Processing Unit; GPU: Graphical Processing Unit; RMSD: Root mean square deviation.

\section{REFERENCES}

1. Cancer.org [homepage on the Internet]. Understanding Genetic Testing for Cancer. American Cancer Society, Inc. [updated 2016; cited 2016 Dec 24]. Available from https://www.cancer.org/cancer/cancer-causes/genetics/understandinggenetic-testing-for-cancer.html.

2. Dickens MP, Fitzgerald R, Fischer PM. Small-molecule inhibitors of MDM2 as new anticancer therapeutics. Semin Cancer Biol. 2010;20(1):10-8.

3. Tian X, Tang H, Lin H, Cheng G, Wang S, Zhang X. Saponins: The potential chemotherapeutic agents in pursuing new anti-glioblastoma drugs. Mini Rev Med Chem. 2013;13(12):1709-24.

4. Huang RM, Yi XX, Zhou Y, Su X, Peng Y, Gao CH. An update on 2, 5-diketopiperazines from marine organisms. Mar Drugs. 2014;12(12):6213-35.

5. Ekins S, Mestres J, Testa B. In silico pharmacology for drug discovery: Methods for virtual ligand screening and profiling. Br J Pharmacol. 2007;152(1):9-20.

6. Hetényi C, Van der Spoel D. Blind docking of drug-sized compounds to proteins with up to a thousand residues. FEBS Lett. 2006;580(5):1447-50.

7. Morris GM, Goodsell DS, Huey R, HartWE, Halliday S, Belew R, et al. Autodock: Automated docking of flexible ligands to receptors. 2001. Available from http:// saf.bio.caltech.edu/hhmi_manuals/autodock/autodock2_4_userguide.pdf.

8. Shahsavand S. BCB410 Protein-Ligand Docking Exercise Set. 2011.

9. Delano WL. PyMOL User Guide. 2004. [cited 2017 Jan 1]. Available from http:// pymol.sourceforge.net/newman/userman.pdf/

10. Wolber G. LigandScout User Manual. 2010. [cited 2016 Dec 11]. Available from http://www.inteligand.com/ligandscout3/downloads/ ligandscout-manual2010-04-15.pdf.

11. Wallace AC, Laskowski RA, Thornton JM. LIGPLOT: A program to generate schematic diagrams of protein-ligand interactions. Protein Eng. 2017;8(2):127-34. 